\title{
Research Paper \\ Effect of White Noise on the Sleep of Elderly Patients Hospitalized in Coronary Care Units
}

\author{
Pouya Farokhnezhad Afshar ${ }^{1},{ }^{*}$ Hosein Zahednezhad², Mehdi Ajri Khamesloo ${ }^{3}$, Reza Ghanei Gheshlagh', Reza Fathi ${ }^{4}$
}

1. Department of Ageing, University of Social Welfare and Rehabilitation Sciences, Tehran, Iran.

2. Department of Nursing, University of Social Welfare and Rehabilitation Sciences, Tehran, Iran.

3. Department of Midwifery \& Fertility Health, School of Nursing and Midwifery, Shahid Beheshti University of Medical Sciences, Tehran, Iran.

4. Department of Nursing, School of Nursing and Midwifery, Tehran University of Medical Sciences, Tehran, Iran.

Citation: Farokhnezhad Afshar P, Zahednezhad H, Ajri Khamesloo M, Ghanei Gheshlagh R, Fathi R. [The effect of white noise on the sleep of elderly patients hospitalized in coronary care units (Persian)]. Iranian Journal of Ageing. 2016; 11(4):44-51. http://dx.doi.org/10.21859/sija-110144

doi): http://dx.doi.org/10.21859/sija-110144

Received: 16 Nov. 2015 Accepted: 18 Jan. 2016

Key words: Elderly patient, Music, Sleep, Coronary care unit

\section{ABSTRACT}

Objectives Sleep disorders are prevalent among elderly patients admitted to coronary care units (CCUs). Moreover, the sleep disorders intensify with age-related changes. Thus, this study aimed to determine the effect of white noise on the sleep of elderly patients admitted to CCUs.

Methods \& Materials This quasi-experimental research included 40 patients who were admitted to CCU. The sampling was done by convenience sampling method and by following the inclusion criteria. In this study, sleep duration and sleep latency of the patients were measured on the first day of hospital admission and 1 week after hospitalization in CCU in both the control group and experimental group. The experimental group received "white noise" (ocean sound) for 2 hours each day before bedtime for 1 week while the control group received no such noise. The obtained data were analyzed by statistical tests (Chi-squared test, independent t-test, and paired t-test) through SPSS, version 18.

Results The mean (SD) age of the participants was 68.75 (6.19) years in the control group and 67.05 (5.15) years in the experimental group. Most participants were men (57.5\%). In addition, most of them were hospitalized with acute coronary syndrome (80\%), and $65 \%$ had a history of hospitalization. The mean (SD) sleep duration in the control group was $7.16(0.86)$ hours before the intervention, which decreased to $4.69 \pm 0.56$ hours after the study. The mean (SD) sleep latency in the control group increased from $20 \pm 5.4$ minutes to $28 \pm 5.4$ minutes after the intervention, which shows a significant reduction in sleep quantity after hospitalization $(\mathrm{P}<0.001)$. The mean (SD) sleep duration of the experimental group did not show any significant difference with that of the control group (6.75 [0.91] h) ( $P=0.146)$. Furthermore, after the study, the mean (SD) of sleep duration in the experimental group did not show any significant reduction (7.04 [0.91] h) ( $\mathrm{P}=0.12)$. The sleep latency duration of the older people in the control group decreased from 19 (5.4) minutes to 17 (4.2) minutes ( $P=0.17)$. The comparison of sleep duration and sleep latency of both groups after the study indicated a significant difference between 2 groups $(P<0.001$ for both variables).

Conclusion Our results showed that white noise did not significantly change the sleep quality of old patients; however, it prevented the decrease in sleep duration and the increase in sleep latency duration. White noise with regard to its effects on increasing auditory stimulation threshold, its sleep induction, and suitable cost-effectiveness, can be used for some old patients hospitalized in CCUs.

\footnotetext{
* Corresponding Author:

Hosein Zahednezhad, PhD Candidate

Address: Department of Nursing, University of Social Welfare and Rehabilitation Sciences, Kodakyar Ave., Daneshjo Blv., Evin, Tehran, Iran Tel: +98 (21) 22180083

E-mail: zahednezhad.h@gmail.com
} 


\title{
تأثير صداى سفيد بر خواب بيمار ان سالمند بسترى در واحد مراقبت كرونرى
}

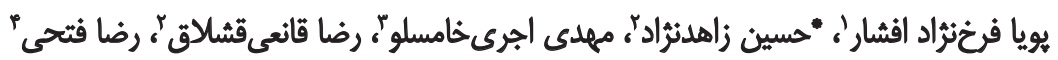

1- كروه سالمندى، دانشَّاه علوم بهزيستى و توانبخشى، تهران، ايران.

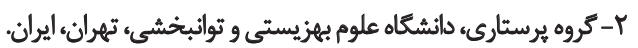

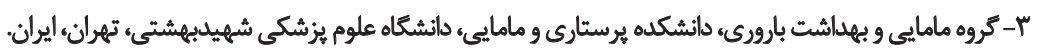

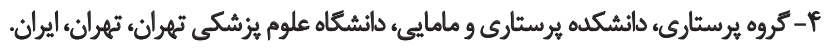

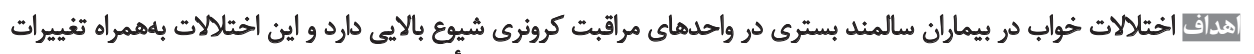

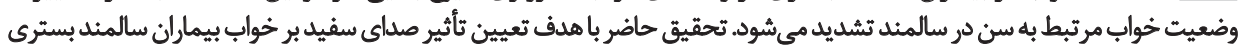

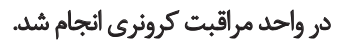

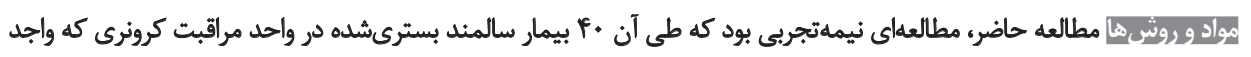

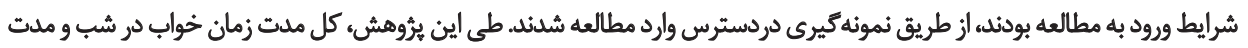

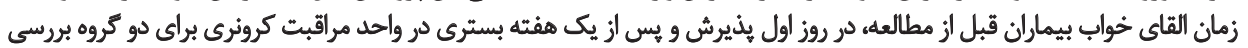

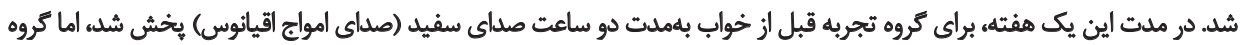

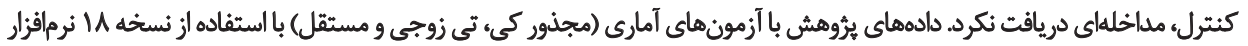
SPSS

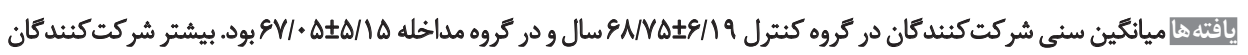

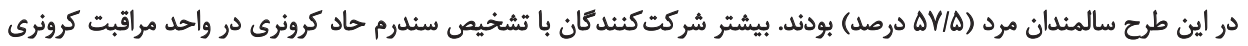

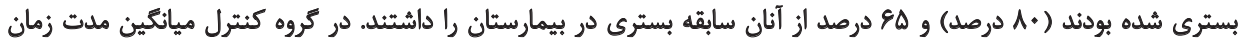

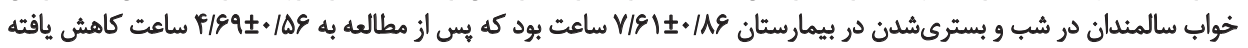

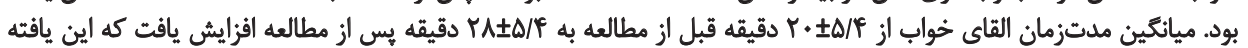

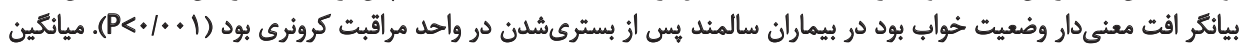

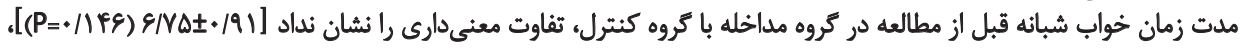

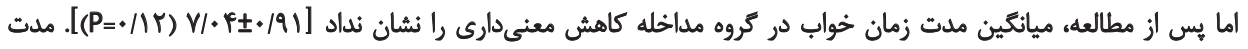

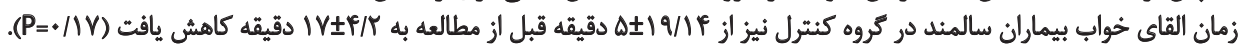

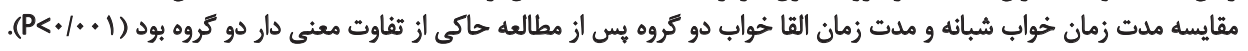

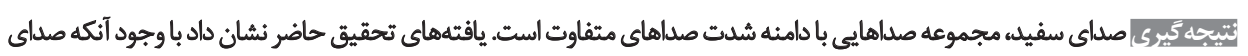

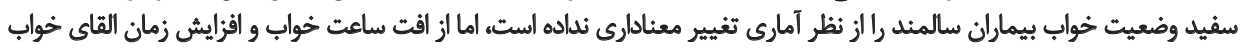

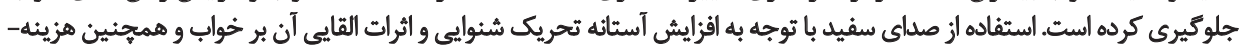

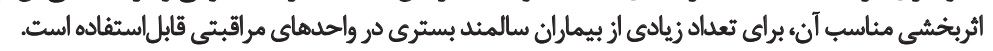

كليدوارهها: بيماران سالمند،

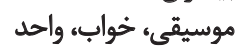
مراقبت كرونرى

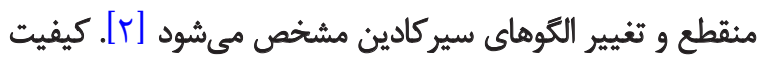

مelo

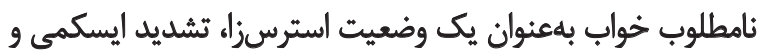
انفاركتوس قلبى را در بيمار ان بهوجود مي آورد [ب]

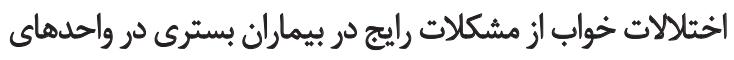

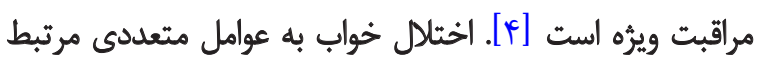
خواب بهعنوان ثعديلكنئده عملكرد قلبى-عروقى در شرايط

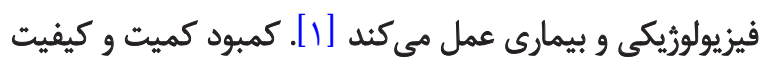
خواب بهصورت كاهش زمان كل خواب، كاهش عمق عي خواب، خواب

$$
\text { - }
$$

* تويسئده مسئول:

حسين زاهدئراد

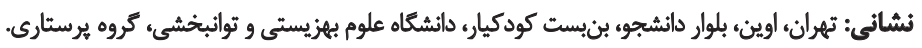

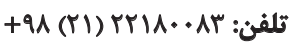
يست الكتروئيكى: تلبن، zahednezhad.h@gmail.com 
مطالعات خواب روى سالمندان نشان دادهاند كه افراد مسن دجار كاهش زمان كل خواب، كاهش كفايت خواب و افزايش زمان بان بيدارى

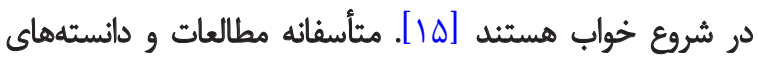

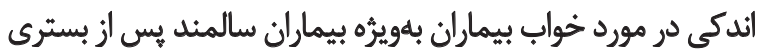
در واحد مراقبت كرونرى وجود دارد [هـ].

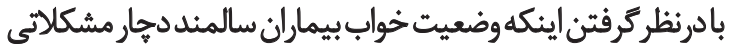

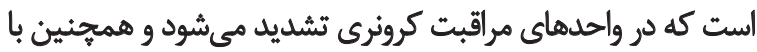

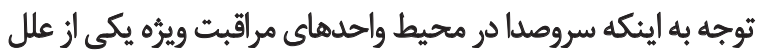

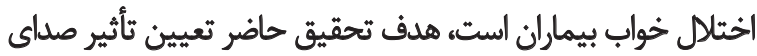

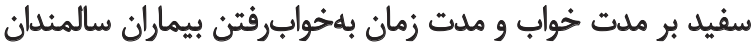

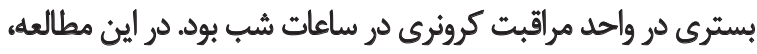

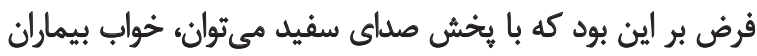
سالمندر ابهببود بخشيد.

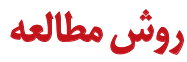

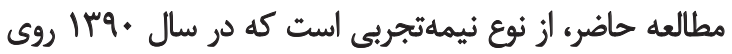

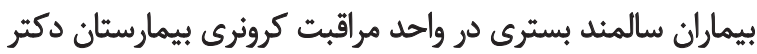

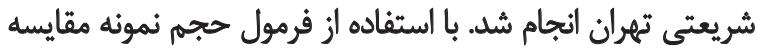

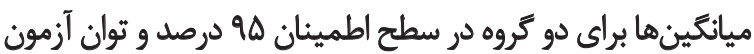

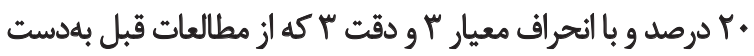

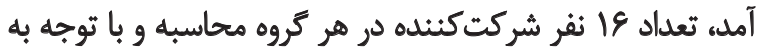

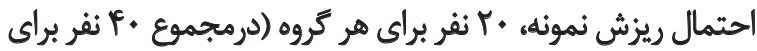

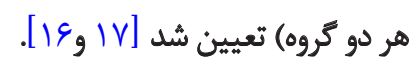

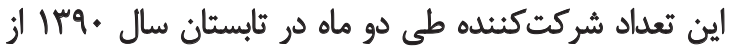

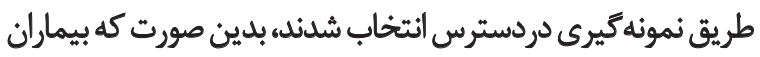

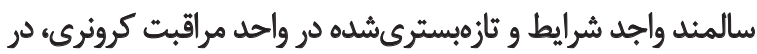

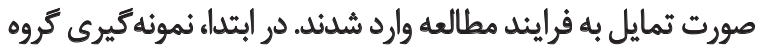

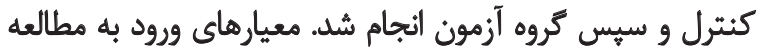

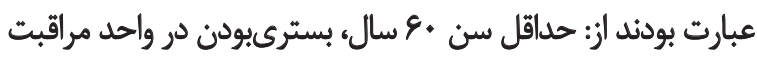

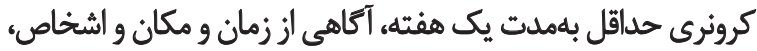

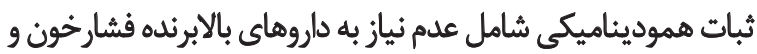

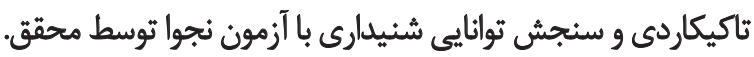

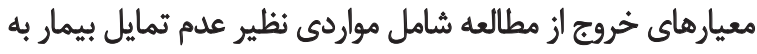

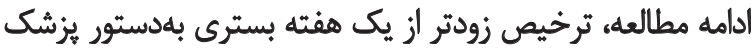
معالج، درد شديد و نياز به احياى قلبى و و ريوى طي مطي مطالعه بودئد.

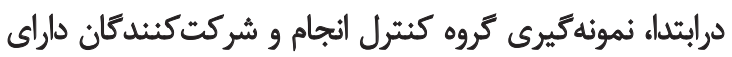

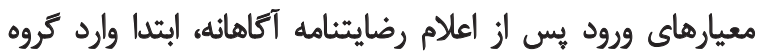

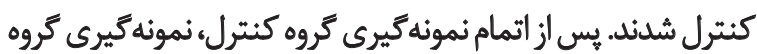

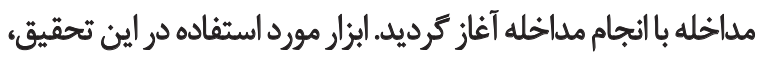

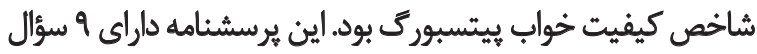

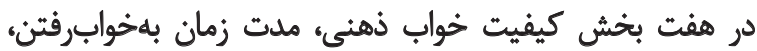
كفايت خواب، مدت زمان خواب، اختلال خواب، استفاده از داروهاى موني
استه اما ميزان تأثير هريك از اين عوامل بهروشنى مشخص نيست

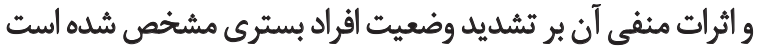

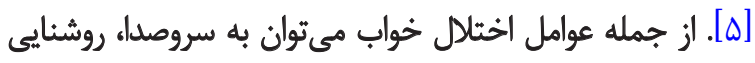

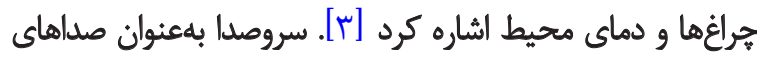

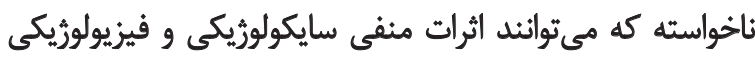

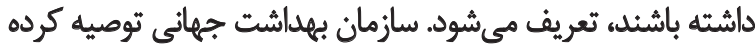

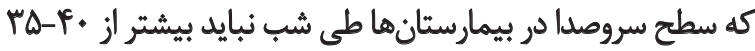

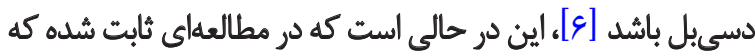

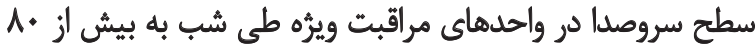

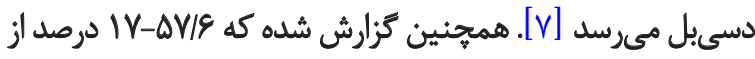
عوامل محرك بيدارى بيماران در واحد مراقبت ويرٔه سروصدا بوردا بوده

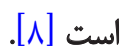

با وجود بسيارى از ادعاها مبنىبر اقدامات كاهنده سروصدا كه

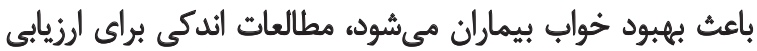

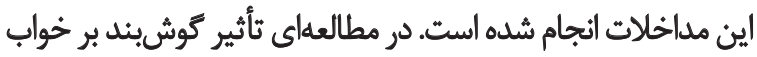

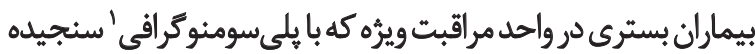

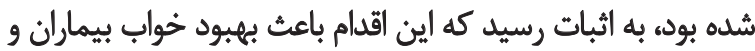

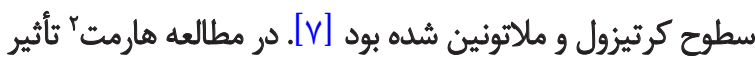
موسيقى كلاسيك آرامبخش روى

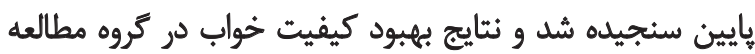

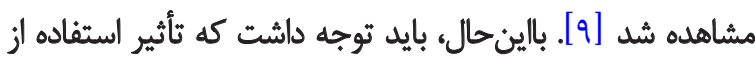

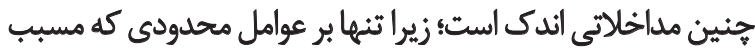
مشكلات خواب هستند، تأثير مي كذارند.

صداى سفيد، صدايى است كه باعث تغيير سطح آستانه تحريك

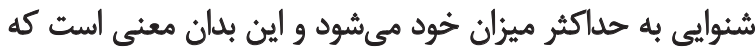

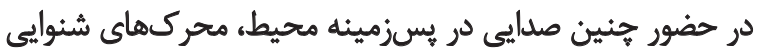

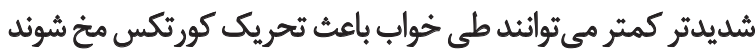

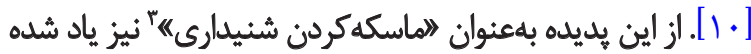

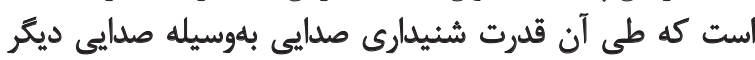

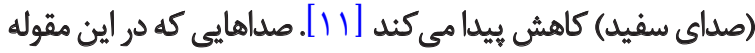

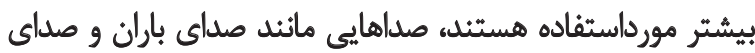

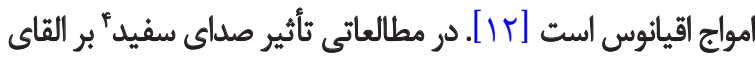

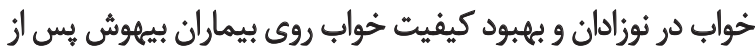

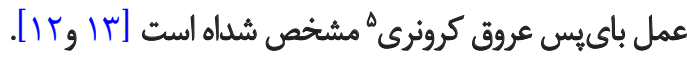
حدود •ه درصد سالمندان، از خواب خود شكايت دارند و اين باين

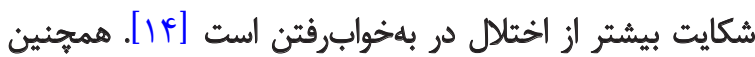

1. Polysomnography

2. Harmat

3. Auditory masking

4. White noise

5. Coronary artery bypass graft 
بسترىشده، قبل خواب از صداي اقيانوس " استفاده شده بود كه

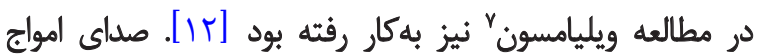

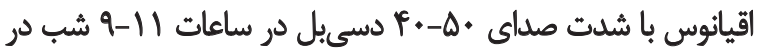

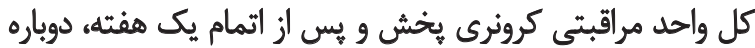

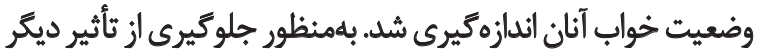

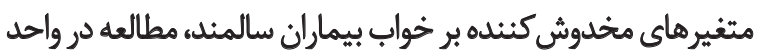
مراقبت كرونرى يكسان و در ساعات يكسان انجام شد.

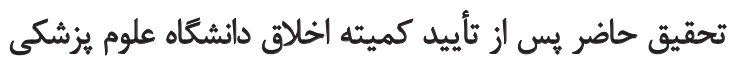

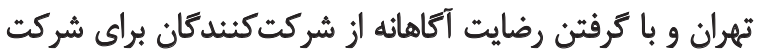

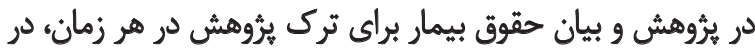

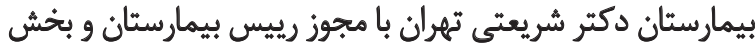

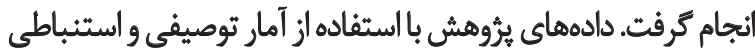
(مجذور كاى و تى زوج و مستقل بهدليل توزيع طبيعى متغيرها) با بادئ نسخه A| نرم|فزار SPSS تجزيه و تحليل شد.

يافتهها

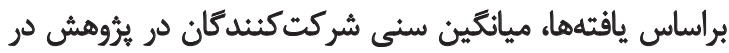
Sروه كنترل \&N/VA

6. Ocean sound

7. Williamson
خوابآور و عملكرد ناقص در طول روز است. هر بخش از بـ- امتياز

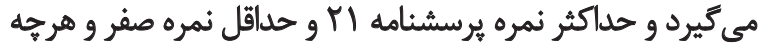

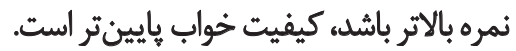

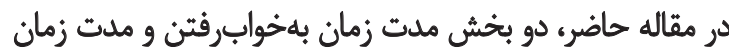

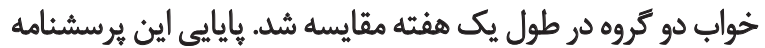

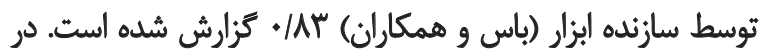

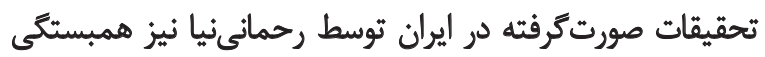

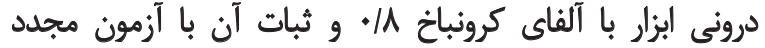

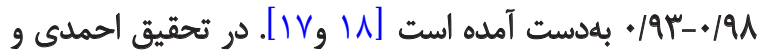

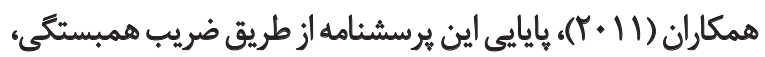

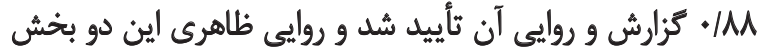

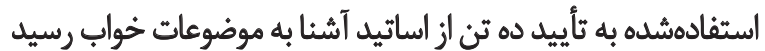

[19]

روش مداخله به اين صورت بود كه در ابتدا، در روز اول بسترى

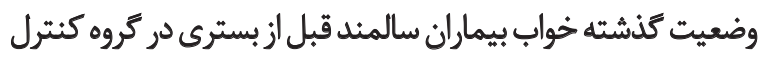

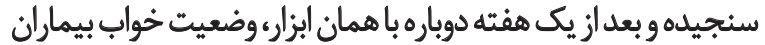

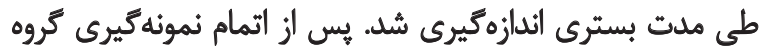

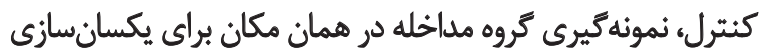

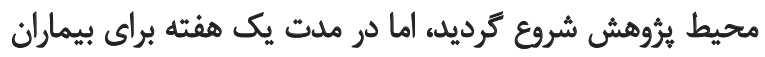

جدول ا. خصوصيات شركت كثندكان بسترى در بخش مراقبت ويُره كروئرى بيمارستان دكتر شريعتى تهران.

\begin{tabular}{|c|c|c|c|c|c|c|}
\hline \multirow{3}{*}{ آزمون أَمارى } & \multicolumn{3}{|c|}{ مداخله } & \multicolumn{2}{|c|}{ كتثرل } & \multirow{2}{*}{ خُوروهيات } \\
\hline & درصد & & & درصد & تعداد & \\
\hline & $1+* \%$ & & & $1++\%$ & $r+$ & جمع \\
\hline \multirow{2}{*}{$P=1$} & $\Delta \Delta$ & 11 & g. & ir & مرد & \multirow{2}{*}{ جنسيت } \\
\hline & PD & 9 & f. & $\wedge$ & زن & \\
\hline \multirow{2}{*}{$P=1$} & A. & if & A. & 18 & سندرم حاد كرونرى & \multirow{2}{*}{ علت بسترى } \\
\hline & r. & f & $r+$ & f & "علل ديكر & \\
\hline \multirow{2}{*}{$\mathrm{P}=+M^{e}$} & \&. & $\pi$ & v. & if & د & \multirow[b]{2}{*}{ سابقه بسترى } \\
\hline & $f_{0}$ & $\Lambda$ & r. & 8 & ثلدارد & \\
\hline \multirow{2}{*}{$\mathrm{P}=\cdot /$ rre } & $\Delta$ & 1 & r. & f & همرد & \multirow{2}{*}{ وضعيث تأهل } \\
\hline & 90 & 19 & A. & 18 & مثأهل & \\
\hline \multirow{3}{*}{$\mathrm{P}=\cdot 18$} & ra & $v$ & TQ & $\Delta$ & شاغل & \multirow{3}{*}{ الشتغال } \\
\hline & TD & $v$ & r. & 8 & بيكار & \\
\hline & r. & 8 & Fa & 9 & بازئشسته & \\
\hline
\end{tabular}


جدول ז. مقايسه ميانكّين مدت زمان خواب شب، قبل و بعد از مداخله در دو كروه كنترل و مداخله بيماران سالمند بسترى در بخش مراقبت ويزٔه كرونرى.

\begin{tabular}{|c|c|c|c|}
\hline $\begin{array}{c}\text { P-value } \\
\text { df }\end{array}$ & مداخله & كتئرل & \\
\hline $\begin{array}{c}\text {. NFe } \\
\text { ra }\end{array}$ & $g / V \Delta \pm+/ q)$ & W/ISE./NE & قبل \\
\hline $\begin{array}{c}<\cdot 1 * .1 \\
r \mu\end{array}$ & $V / \cdot f \pm \cdot / 9)$ & $p / g q \pm \cdot / \Delta q$ & بعد \\
\hline & $\begin{array}{c}. / 14 \\
19\end{array}$ & $\begin{array}{c}<+1++1 \\
19\end{array}$ & $\begin{array}{c}\text { P-value } \\
\text { df }\end{array}$ \\
\hline
\end{tabular}

L

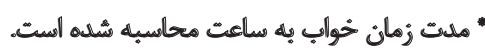

جدول "٪. مقايسه ميانكين مدت زمان القاى خواب، قبل و بعد از مداخله در دو كروه كنترل و مداخله بيماران سالمند بسترى در بخش مراقبت ويزٔه كرونرى.

\begin{tabular}{|c|c|c|c|}
\hline $\begin{array}{c}\text { P-value } \\
\text { df }\end{array}$ & مداخله & $\begin{array}{l}\text { كترل } \\
\text { XISD }\end{array}$ & \\
\hline $\begin{array}{l}.19 V r \\
\text { HA }\end{array}$ & $. / 19 \pm . / .9$ & $. / r+ \pm . / .9^{\circ}$ & قبل \\
\hline $\begin{array}{c}<\cdot 1 \cdot .1 \\
\mathrm{H}\end{array}$ & $. / V \pm \pm . / \vee V$ &.$/$ rA土./.9 & بعد \\
\hline & $\begin{array}{l}.118 \\
19\end{array}$ & $\begin{array}{c}<+1 \cdot+1 \\
19\end{array}$ & $\begin{array}{c}\mathrm{P} \text {-value } \\
\mathrm{df}\end{array}$ \\
\hline
\end{tabular}

كالم

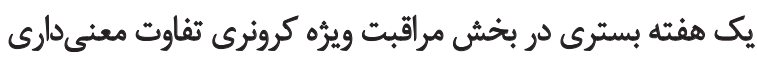

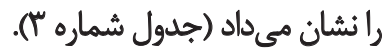

ث

وضعيت خواب قبل از مطالعه نسبت به بعد از مداخله، در كروه

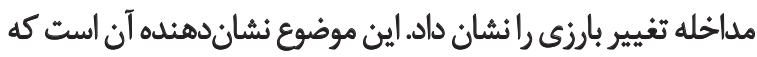

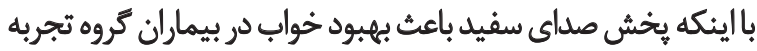

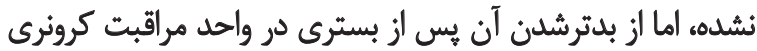

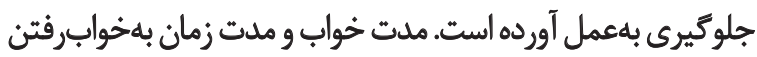

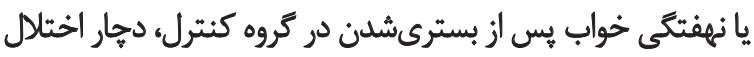

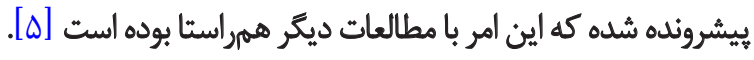
با توجه به مشكلات خواب كه در سنين سالمندى شيوع نسبتاً

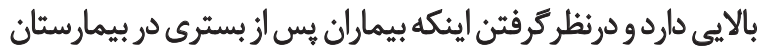

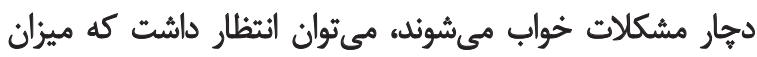
مشكلات خواب در بيماران سالمند بسترى در بيمارستان بيشتر مئر

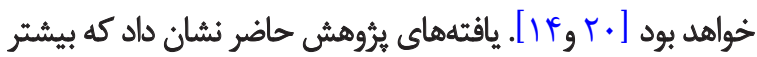

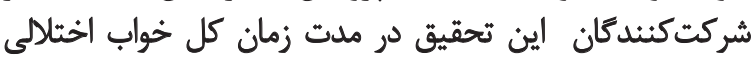

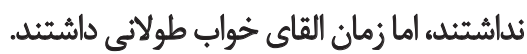

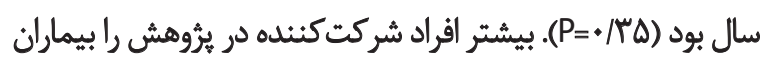

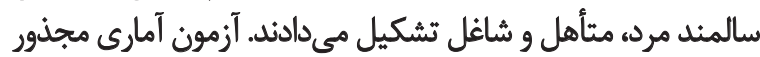

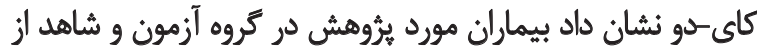

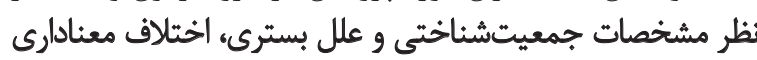
نداشتند (جدول شماره ()). مقايسه ميانكين مدت زمان خواب (به ساعت) بيماران سالمند

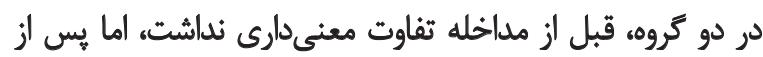

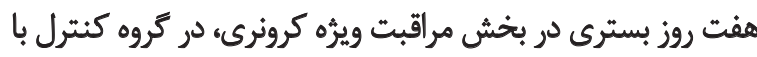

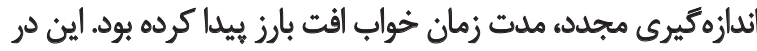

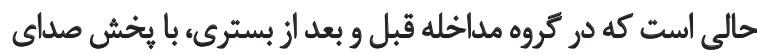

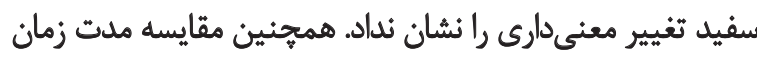

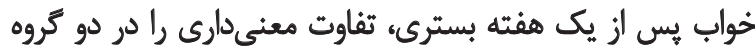

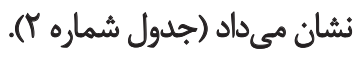
مقايسه ميانتين مدت زمان بهخوابرفتن (به دقيقه) در دو زروه،

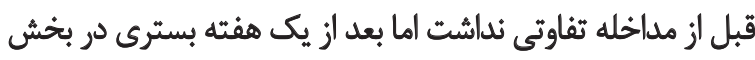

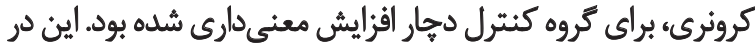

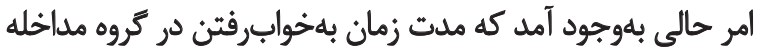

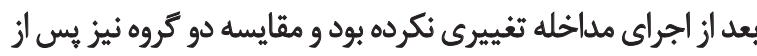


كه در انجام اين مطالعه محققان را يارى نمودند و نيز از كاركنان

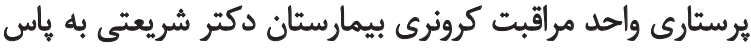
زحماتشان كمال تشكر و قدردانى بهممل مى بيماريد
از جمله علل همهم براى بروز اختلالات خواب، سروصداى

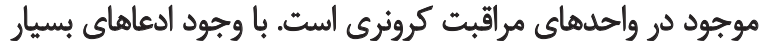

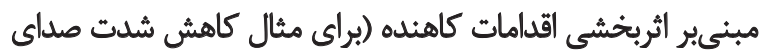

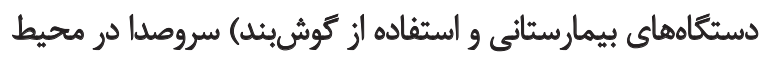

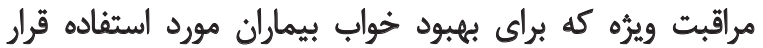

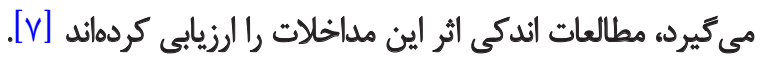

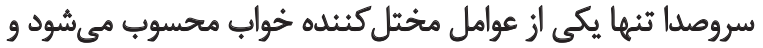

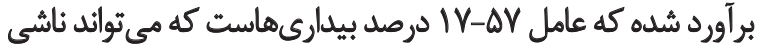

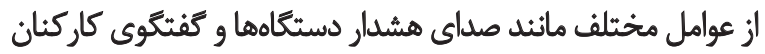

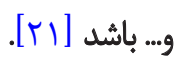

يافتههاى تحقيق يس از مداخله در گروه كنترل نشان داد كه

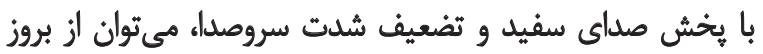
اختلالات خواب جلوكيرى كرد.

بالينحال، بايد توجه داشت كه شرايط محيطى واحدهاى مراقبت نوايت

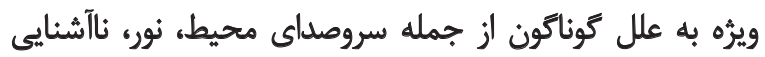

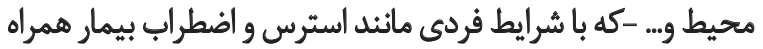

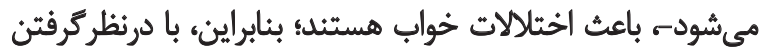

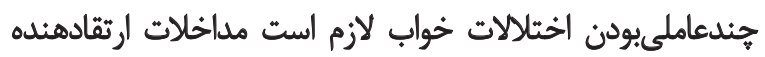

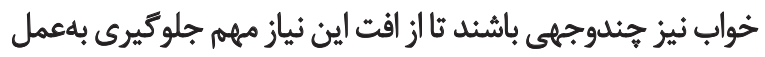

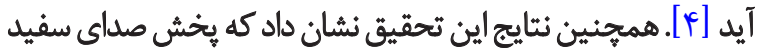

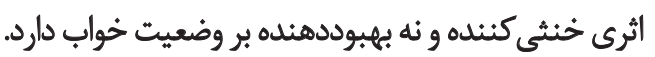

\section{تتيجه}

تحقيق حاضر با هدف بررسى تأثير صداي سفيد بر خواب بيماران

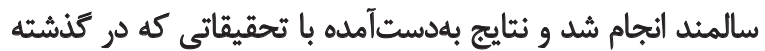

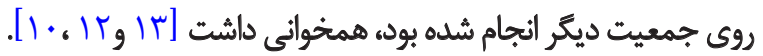

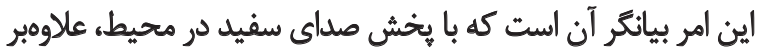

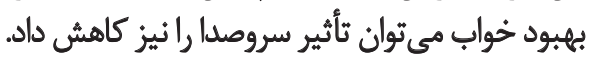

از جمله محدوديتهاي اين برؤوهش مي توان به ناثواني در كنترل

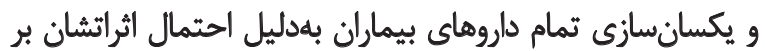

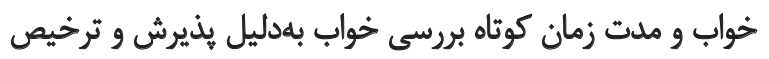

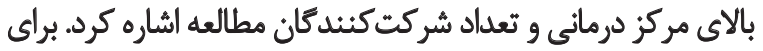

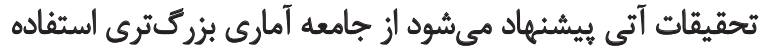

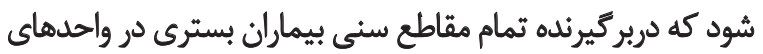
مراقبت ويرٔه باشد.

$$
\text { تشكر و قدرداثي }
$$

اين مقاله حاصل بخشى از طرح تحقيقاتى مصوب مركز تحقيقات

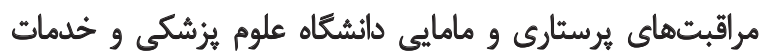
بهداشتى درمانى تهران با شماره

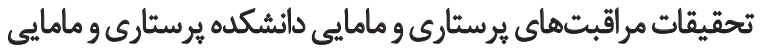

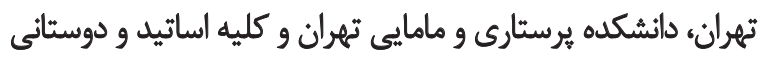




\section{References}

[1] Wolk R, Gami A, Garcia T, Somers V. Sleep and cardiovascular disease. Current Problems in Cardiology. 2005; 30(12):625-62.

[2] Hardin K. Sleep in the ICU Potential Mechanisms and Clinical Implications. Chest Journal. 2009; 136(1):284-94

[3] Fontana CJ, Pittiglio LI. Sleep deprivation among critical care patients. Critical Care Nursing Quarterly. 2010; 33(1):75-81.

[4] Zolfaghari M, Farokhnezhad, Afshar P, Asadi Noghabi A, Ajri Khameslou M. [Modification of Environmental Factors on Quality of Sleep among Patients Admitted to CCU (Persian)]. Hayat. 2012; 18(4):61-8.

[5] Schiza S, Simantirakis E, Bouloukaki I, Mermigkis C, Arfanakis D, Chrysostomakis S, et al. Sleep patterns in patients with acute coronary syndromes. Sleep Medicine. 2010; 11(2):149-53.

[6] Freedman NS, Kotzer N, Schwab RJ. Patient perception of sleep quality and etiology of sleep disruption in the intensive care unit. American Journal of Respiratory and Critical Care Medicine. 1999; 159(4):1155-62.

[7] Hu RF, Jiang XY, Zeng YM, Chen XY, Zhang YH. Effects of earplugs and eye masks on nocturnal sleep, melatonin and cortisol in a simulated intensive care unit environment. Critical Care. 2010; 14(2):66.

[8] Xie H, Kang J, Mills GH. Clinical review: The impact of noise on patients' sleep and the effectiveness of noise reduction strategies in intensive care units. Critical Care. 2009; 13(2):208.

[9] Harmat L, Takács J, Bodizs R. Music improves sleep quality in students. Journal of Advanced Nursing. 2008; 62(3):327-35.

[10] Stanchina ML, Abu-Hijleh M, Chaudhry BK, Carlisle CC, Millman RP. The influence of white noise on sleep in subjects exposed to ICU noise. Sleep Medicine. 2005; 6(5):423-8

[11] Nakasato N, Kumabe T, Kanno A, Ohtomo S, Mizoi K, Yoshimoto T. Neuromagnetic evaluation of cortical auditory function in patients with temporal lobe tumors. Journal of Neurosurgery. 1997; 86(4):610-8

[12] Williamson J. The effects of ocean sounds on sleep after coronary artery bypass graft surgery. American Journal of Critical Care. 1992; 1(1):91-7.

[13] Spencer JA, Moran DJ, Lee A, Talbert D. White noise and sleep induction. Archives of Disease in Childhood. 1990; 65(1):135-7.

[14] Maghsoudnia S. [Primary health care for old adulta in Iran (Persian)]. $2^{\text {nd }} \mathrm{ed}$. Tehran: University of Social Welfare And Rehabilitation Sciences; 2006.

[15] Ohayon MM, Vecchierini M. Normative sleep data, cognitive function and daily living activities in older adults in the community. Sleep. 2005; 28(8):981-89.

[16] Abolhasani S. [Evaluation of sensory stimulation on sleep deprivation symptoms in patients admitted to coronary care unit (Persian)]. Journal of Semnan University of Medical Sciences. 2005; 7(1):34-5.

[17] Rahmani nia F, Mohebi H, Brojeni M. [Effects of walking on the quality and quantity of sleep and sleep-related physiological parameters in elderly men (Persian)]. Journal of Sport Biosciences. 2009; 1 (3):111- 26
[18] Izadi Avanji F, Adib Hajbaghery M, Afazel M. [Quality of sleep and its related factors in the hospitalized elderly patients of Kashan hospitals (Persian)]. Feyz. 2009; 12(4):52-9.

[19] Ahmadi S, Khankeh HR, Mohammadi F, Fallahi M, Reza Soltani P. [The effect of sleep restriction treatment on quality of sleep in the elders (Persian)]. Iranian Journal of Ageing. 2011; 5(1):7-15.

[20] Kazemi M, Rafiee G, Ansari A. [Factors related to sleep disturbance in patients admitted to hospital and surgical wards of Imam Ali ibn AbiTalib (Persian)]. Journal of Rafsanjan University of Medical Sciences. 2005; 4(4):270-5

[21] Lawson N, Thompson K, Saunders G, Saiz J, Richardson J, Brown D, et al. Sound intensity and noise evaluation in a critical care unit. American Journal of Critical Care. 2010; 19(6):88-98. 
